\title{
Gauge independence of magnetic moment and vanishing charge of Dirac neutrinos: an exact one-loop demonstration
}

\author{
Wen-Tao Hou ${ }^{a, b}$, Yi Liao 1 1, and Hong-Jun Liu ${ }^{a}$ \\ a School of Physics, Nankai University, Tianjin 300071, China \\ ${ }^{b}$ Department of Modern Physics, University of Science and Technology of China, \\ Hefei 230026, Anhui, China
}

\begin{abstract}
The magnetic moment and vanishing charge of a Dirac neutrino are physically observable quantities and must not depend on the choice of gauge in a consistent quantum field theory. We verify this statement explicitly at the one loop level in both $R_{\xi}$ and unitary gauges of the minimally extended standard model. We accomplish this by manipulating directly the integrands of loop integrals and employing simple algebraic identities and integral relations. Our result generally applies for any masses of the relevant particles and unitary neutrino mixing.
\end{abstract}

PACS: 14.60.Lm, 13.40.Em, 12.15.Lk

\footnotetext{
${ }^{1}$ liaoy@nankai.edu.cn
} 


\section{Introduction}

The fundamental properties of a particle like its charge and electromagnetic dipole moments are physical quantities that in principle are experimentally measurable, for reviews, see Refs. [1, 2]. These quantities can be unambiguously calculated in a quantum-mechanically consistent theory like the standard model (SM), and confronted with the measurements to decide whether the theory is correct or not. That said, the practical calculation and demonstration of its result being independent of computational methods are not always trivial. We have witnessed a similar circumstance recently, concerning the one-loop contribution of the charged weak gauge bosons $W^{ \pm}$to the two-photon decay rate of the Higgs boson. A new computation in unitary gauge [3, 4] claimed an answer that is different from the well-spread result obtained long ago [5, 6, 7, 8] in a special gauge, i.e., the 't Hooft-Feynman gauge ( $\xi=1)$ among the class of renormalizable $R_{\xi}$ gauges. Subsequent studies by various methods, including computing in both $R_{\xi}$ and unitary gauges, see for instance Refs. [9, 10], confirmed the old result, and taught us a great deal on computational subtleties in a theory that is nontrivial in the high energy regime.

In this work we examine a similar problem in the neutrino sector, i.e., the charge and magnetic moment of a Dirac neutrino in SM that is minimally extended by the introduction of right-handed neutrinos. We show explicitly at the one-loop level in both $R_{\xi}$ and unitary gauges that the neutrino charge vanishes and its magnetic moment is a gauge independent quantity. The issue has been partially studied in the literature. Early works [11, 12, 13, 14, 15] assumed a massless neutrino or expanded the quantities to the leading order in the small masses of neutrinos and charged leptons, ignored the lepton mixing, or computed in a special gauge. A further step was taken some years ago [16, 17, 18]. It was found [17], for instance, that up to the second order in the expansion of small neutrino masses the charge vanishes and the magnetic moment is $\xi$-independent, and that the charge vanishes exactly in the 't Hooft-Feynman gauge. Here we cope directly with the integrands of loop integrals, and demonstrate manifestly that both quantities are gauge independent for any masses of the relevant particles and for any unitary lepton mixing.

In the next section we set up our notations and suggest how to calculate in a nice way to isolate the terms that potentially contribute to the charge and magnetic moment. We describe in some detail in sec 3 our calculation in $R_{\xi}$ gauge. Our one-loop exact result for the magnetic form factor at vanishing momentum transfer is shown in Eq. (59). This is followed by a short discussion in sec 4 on the calculation in unitary gauge. We summarize briefly in the last section.

\section{Computational strategy}

The charge and magnetic moment of a Dirac particle can be defined by the amplitude of a process in which it radiates a photon,

$$
\bar{u}\left(p_{-}\right) i \mathscr{A}_{\mu}(q) u\left(p_{+}\right)=(-i e) \bar{u}\left(p_{-}\right)\left[\gamma_{\mu} F_{1}\left(q^{2}\right)-\frac{1}{2 m} i \sigma_{\mu \nu} q^{v} F_{2}\left(q^{2}\right)+\cdots\right] u\left(p_{+}\right) .
$$


Here $p_{ \pm}=p \pm q / 2$ are the momenta of the incoming and outgoing particle of mass $m$, and $q$ is the photon's outgoing momentum. The above decomposition in terms of the standard form factors is based on Lorentz covariance and electromagnetic gauge invariance, and assumes that the Dirac particle in both initial and final states is physical:

$$
\not p_{ \pm} u\left(p_{ \pm}\right)=m u\left(p_{ \pm}\right), p_{ \pm}^{2}=m^{2}
$$

The dots in Eq. (1) stand for two more form factors that are irrelevant here; one corresponds to the electric dipole moment that cannot occur at one loop in the minimally extended SM (as can also be seen from sec 3), and the other is the so-called anapole whose Lorentz structure is quadratic in $q$. The form factors at an arbitrary $q^{2}$ are generally not measurable quantities, since the above (unphysical) amplitude appears as part of the complete contribution to a physical process. Nevertheless, $F_{1}(0)$ and $F_{2}(0)$ are physical quantities because they correspond to the charge and anomalous magnetic moment of the particle. Our convention is such that the electron has the charge $e F_{1}(0)=e<0$ and the magnetic moment vector, $\vec{\mu}=(e / m)\left[F_{1}(0)+F_{2}(0)\right] \vec{S}$ with $\vec{S}$ being its spin vector, that appears, e.g., in the interaction potential of the dipole with an external magnetic field $\vec{B}, V=-\vec{\mu} \cdot \vec{B}$.

The charge, $F_{1}(0)$, is relatively easy to isolate. Setting $q=0$ removes all other Lorentz structures, and allows us to employ the equations of motion (EoMs) in the limit $q \rightarrow 0$ for both initial and final particles, $\not p u(p)=m u(p)$, to reduce the amplitude completely to the $\gamma_{\mu}$ form. There are several ways to work out the anomalous magnetic moment $F_{2}(0)$. One could isolate by brute force terms contributing to the form factor $F_{2}\left(q^{2}\right)$ and take its value at $q^{2}=0$. Most studies in the literature follow this approach. In the second approach, one employs a projection operator, and expresses $F_{2}(0)$ as a combination of Dirac traces [19, 20, 21]. Here we take a third approach, which might be the best to observe the cancellation of gauge dependence among various Feynman graphs. As we will show in the next section, the cancellation happens at the level of loop integrands. In this approach, we take the derivative of the amplitude with respect to the photon momentum, $i \partial_{\nu}^{q} \mathscr{A}_{\mu}(q)$, antisymmetrize it in the Lorentz indices $\mu$ and $v$, and then evaluate it at $q=0$. Since all form factors are smooth at $q^{2}=0$, only the magnetic moment term survives the procedure and yields $-e /(2 m) \sigma_{\mu \nu} F_{2}(0)$. [We remind once again that the electric dipole term vanishes at one loop but would appear at higher orders.] Comparison of the two gives the answer for $F_{2}(0)$.

An important point in implementing the above procedure should be noted. We mentioned that the decomposition in eq (1) is possible only upon using EoMs (2). When computing $F_{2}(0)$, we are essentially expanding $\mathscr{A}_{\mu}(q)$ in small $q$ and isolating its linear terms. A term that is manifestly linear in $q$ cannot avoid our eyes, for which we are free to apply the limiting EoMs, $\not p u(p)=m u(p)$, because the difference to the exact ones does not affect $F_{2}(0)$. With terms of apparently zeroth order in $q$ we should be careful. For these terms, when necessary, we must apply the exact equations (2) since the difference now is exactly what we are interested in and may enter $F_{2}(0)$. Ignoring this will result in an incorrect, gauge-dependent answer. Another point is more technical. Although antisymmetrization in Lorentz indices is not mandatory since it will come out automatically upon finishing the calculation, one can simplify the algebra by doing antisymmtrization at an early stage. 


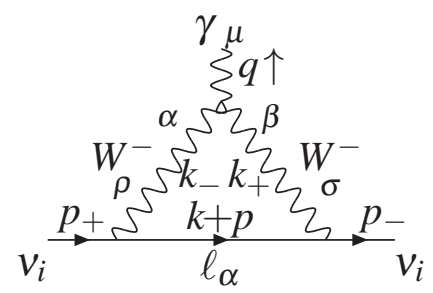

(a)

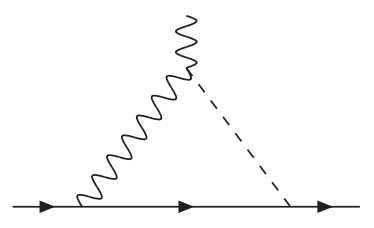

(d)

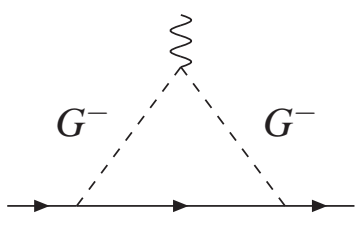

(b)

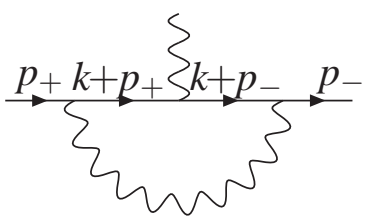

(e)

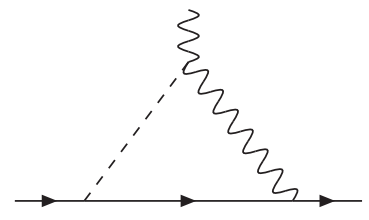

$(c)$

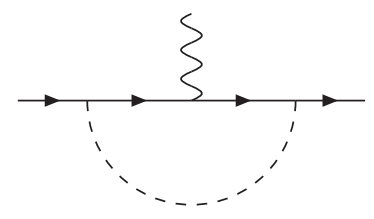

$(f)$

Fig. 1 Feynman diagrams contributing at one loop to the vertex function $i \Gamma_{\mu}(q)$. Wavy (dashed, dotted, solid) lines stand for the gauge boson (scalar, ghost, fermion) fields.

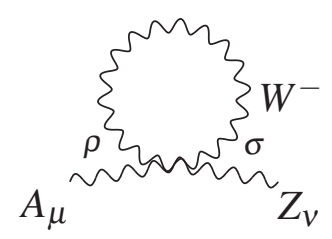

(a)

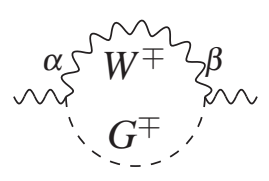

$(e)$

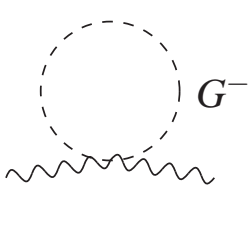

(b)

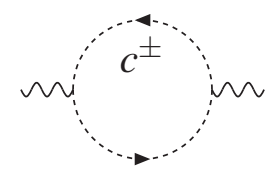

$(f)$

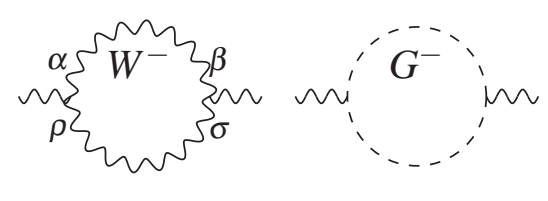

$(c)$

$(d)$

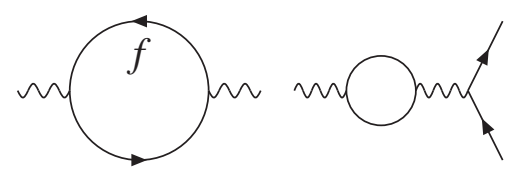

$(g)$

Fig. 2 Feynman diagrams contributing at one loop to the $\gamma Z$ mixing energy $i \Pi_{\mu v}(q)$.

There are two classes of Feynman graphs in SM that contribute at one loop to the amplitude $i \mathscr{A}_{\mu}(q)$, through the proper vertex $i \Gamma_{\mu}(q)$ in Fig. 1 and the photon- $Z$ boson mixing energy in Fig. 2, $i \Pi_{\mu v}(q)$, attached to the tree level neutrino- $Z$ vertex (see the last graph in Fig. 2). While the former contributes to both $F_{1}(0)$ and $F_{2}(0)$, the latter contributes only to $F_{1}(0)$ through

$$
i \Pi_{\mu v}(0) \frac{i}{m_{Z}^{2}} \frac{i g_{2}}{2 c_{W}} \gamma^{v} P_{L} .
$$

Here we use the standard notations of SM: $m_{W, Z}$ are the masses of the $W^{ \pm}$and $Z$ bosons, $g_{2}$ is the gauge coupling of $S U(2)_{L}, c_{W}=\cos \theta_{W}$ and $s_{W}=\sin \theta_{W}$ with $\theta_{W}$ being the weak mixing angle, and $P_{L, R}=\left(1 \mp \gamma_{5}\right) / 2$. We display here the contributions from individual graphs. Working in $d$-dimensions, we write

$$
\begin{aligned}
i \Gamma_{\mu}(q) & =\frac{1}{2} e g_{2}^{2}\left|V_{\alpha i}\right|^{2} \sum_{x=a}^{f} \int_{k}(1 x), \\
i \Pi_{\mu \nu}(0) & =\frac{e g_{2}}{c_{W}} \sum_{x=a}^{f} \int_{k}(2 x), \int_{k} \equiv \int \frac{d^{d} k}{(2 \pi)^{d}},
\end{aligned}
$$


where, denoting $k_{ \pm}=k \pm q / 2$, from Fig. 1,

$$
\begin{aligned}
(1 a) & =+\gamma_{\sigma} P_{L}\left(\not k+\not b+m_{\alpha}\right) \gamma_{\rho} P_{L} \Gamma_{\alpha \beta \mu}\left(-k_{-}, k_{+},-q\right) P^{\alpha \rho}\left(k_{-}\right) P^{\beta \sigma}\left(k_{+}\right) P^{-1}, \\
(1 b) & =+m_{W}^{-2} \frac{\left(m_{i} P_{L}-m_{\alpha} P_{R}\right)\left(\not k+\not p+m_{\alpha}\right)\left(m_{i} P_{R}-m_{\alpha} P_{L}\right)\left(k_{-}+k_{+}\right) \mu}{\left(k_{+}^{2}-\xi_{W} m_{W}^{2}\right)\left(k_{-}^{2}-\xi_{W} m_{W}^{2}\right) P}, \\
(1 c) & =+\frac{\gamma_{\sigma} P_{L}\left(\not k+\not b+m_{\alpha}\right)\left(m_{i} P_{R}-m_{\alpha} P_{L}\right) P^{\mu \sigma}\left(k_{+}\right)}{\left[k_{-}^{2}-\xi_{W} m_{W}^{2}\right] P}, \\
(1 d) & =+\frac{\left(m_{i} P_{L}-m_{\alpha} P_{R}\right)\left(\not k+\not b+m_{\alpha}\right) \gamma_{\rho} P_{L} P^{\mu \rho}\left(k_{-}\right)}{\left[k_{+}^{2}-\xi_{W} m_{W}^{2}\right] P}, \\
(1 e) & =-\frac{\gamma_{\sigma} P_{L}\left(\not k+\not p_{-}+m_{\alpha}\right) \gamma_{\mu}\left(\not k+\not p_{+}+m_{\alpha}\right) \gamma_{\rho} P_{L} P^{\rho \sigma}(k)}{\left[\left(k+p_{+}\right)^{2}-m_{\alpha}^{2}\right]\left[\left(k+p_{-}\right)^{2}-m_{\alpha}^{2}\right]}, \\
(1 f) & =+m_{W}^{-2} \frac{\left(m_{i} P_{L}-m_{\alpha} P_{R}\right)\left(\not k+\not p-+m_{\alpha}\right) \gamma_{\mu}\left(\not k+\not p_{+}+m_{\alpha}\right)\left(m_{i} P_{R}-m_{\alpha} P_{L}\right)}{\left[\left(k+p_{+}\right)^{2}-m_{\alpha}^{2}\right]\left[\left(k+p_{-}\right)^{2}-m_{\alpha}^{2}\right] Q_{2}},
\end{aligned}
$$

and from Fig. 2,

$$
\begin{aligned}
& (2 a)=-c_{W}^{2}\left[2 g_{\rho \sigma} g_{\mu \nu}-g_{\rho \mu} g_{\sigma v}-g_{\rho \nu} g_{\sigma \mu}\right] P^{\rho \sigma}, \\
& (2 b)=-\left(c_{W}^{2}-s_{W}^{2}\right) g_{\mu \nu} Q_{2}^{-1}, \\
& (2 c)=+c_{W}^{2} \Gamma_{\rho \alpha \mu}(-k, k, 0) \Gamma_{\beta \sigma v}(-k, k, 0) P^{\alpha \beta} P^{\rho \sigma}, \\
& (2 d)=+2\left(c_{W}^{2}-s_{W}^{2}\right) k_{\mu} k_{v} Q_{2}^{-2}, \\
& (2 e)=+2 s_{W}^{2} m_{W}^{2} P_{\mu v} Q_{2}^{-1}, \\
& (2 f)=-2 c_{W}^{2} k_{\mu} k_{v} Q_{2}^{-2} .
\end{aligned}
$$

Note that the fermion loop in Fig. 2(g) is transverse and drops out at $q=0$. We have defined the shortcuts for the propagators and triple-gauge vertex:

$$
\begin{aligned}
& \Gamma_{\alpha \beta \mu}\left(p_{1}, p_{2}, p_{3}\right)=\left(p_{2}-p_{3}\right)_{\alpha} g_{\beta \mu}+\left(p_{3}-p_{1}\right)_{\beta} g_{\mu \alpha}+\left(p_{1}-p_{2}\right)_{\mu} g_{\alpha \beta}, \\
& P_{\mu \nu}(p)=g_{\mu \nu}\left[p^{2}-m_{W}^{2}\right]^{-1}-\delta_{W} p_{\mu} p_{v}\left[p^{2}-\xi_{W} m_{W}^{2}\right]^{-1}\left[p^{2}-m_{W}^{2}\right]^{-1} \\
& P=(k+p)^{2}-m_{\alpha}^{2}, Q_{1}=k^{2}-m_{W}^{2}, Q_{2}=k^{2}-\xi_{W} m_{W}^{2}
\end{aligned}
$$

with $P_{\alpha \beta}=P_{\alpha \beta}(k)$ and $\delta_{W}=1-\xi_{W} \cdot m_{\alpha}\left(m_{i}\right)$ is the mass of the charged lepton $\ell_{\alpha}$ (neutrino $v_{i}$ ), and $V_{\alpha i}$ is the lepton mixing matrix appearing in the charged current interaction. A summation over all $\ell_{\alpha}$ is always implied. The identical initial and final neutrino satisfies EoMs (2) where now $m=m_{i}$. The above loop integrands will be manipulated in the next two sections.

\section{Evaluation in $R_{\xi}$ gauge}

\subsection{Charge}

Let us start with the charge. Setting $q=0$ simplifies significantly the expressions of $(1 x)$. Using $(\not k+\not b) \gamma_{\mu}(\not k+\not k)=-(k+p)^{2} \gamma_{\mu}+2(k+p)_{\mu}(\not k+\not b), \partial_{\mu} Q_{2}^{-1}=-2 k_{\mu} Q_{2}^{-2}$, and $\partial_{\mu} P^{-1}=$ $-2(k+p)_{\mu} P^{-2},(1 b)$ and $(1 f)$ sum to a total derivative:

$$
[(1 b)+(1 f)]_{0}=-m_{W}^{-2} \partial_{\mu}\left\{\left[(\not l+\not p)\left(m_{i}^{2} P_{R}+m_{\alpha}^{2} P_{L}\right)-m_{i} m_{\alpha}^{2}\right]\left(P Q_{2}\right)^{-1}\right\},
$$


where the subscript 0 denotes evaluation at $q=0$. Considering the relation

$$
\Gamma_{\alpha \beta \mu}(-k, k, 0) P^{\alpha \rho} P^{\beta \sigma}=\left(k^{\rho} P_{\mu}^{\sigma}+k^{\sigma} P_{\mu}^{\rho}\right) Q_{2}^{-1}+\partial_{\mu} P^{\rho \sigma}
$$

we combine the pure $W^{ \pm}$-loop graphs,

$$
\begin{aligned}
& {[(1 a)+(1 e)]_{0}=+\partial^{\mu}\left\{\gamma_{\sigma}(\not k+\not p) \gamma_{\rho} P_{L} P^{-1} P^{\rho \sigma}\right\}} \\
& +P_{R} \gamma_{\sigma}(\not k+\not \not) \not k P^{\sigma \mu}\left(P Q_{2}\right)^{-1}+\not k(\not k+\not b) \gamma_{\rho} P_{L} P^{\rho \mu}\left(P Q_{2}\right)^{-1} \text {. }
\end{aligned}
$$

The last two terms in the above are summed with the remaining two graphs to yield

$$
\begin{aligned}
& {[(1 a)+(1 e)+(1 c)+(1 d)]_{0}=+\partial^{\mu}\left\{\gamma_{\sigma}(\not k+\not b) \gamma_{\rho} P_{L} P^{-1} P^{\rho \sigma}\right\}} \\
& \left.+P_{R} \gamma_{\sigma}\left[(\not \not+\not p)\left(\not k+m_{i}\right)-m_{\alpha}^{2}\right)\right] P^{\mu \sigma}\left(P Q_{2}\right)^{-1} \\
& +\left[\left(\not k+m_{i}\right)(\not k+\not b)-m_{\alpha}^{2}\right] \gamma_{\rho} P_{L} P^{\mu \rho}\left(P Q_{2}\right)^{-1} \text {. }
\end{aligned}
$$

Since the above expression is sandwiched between the spinors of the initial and final states, it is tempting to replace $m_{i}$ in the last two terms by $\not p$. But this is not legitimate as emphasized in the last section. Instead, $m_{i}$ should be replaced by $(\not p \pm \not / / 2)$ on the rightmost (leftmost), in terms of the exact EoMs (2):

$$
\begin{aligned}
& \bar{u}\left(p_{-}\right)[(1 a)+(1 e)+(1 c)+(1 d)]_{0} u\left(p_{+}\right) \\
= & \bar{u}\left(p_{-}\right)\left(\partial^{\mu}\left\{\gamma_{\sigma}(\not k+\not p) \gamma_{\rho} P_{L} P^{-1} P^{\rho \sigma}\right\}+2 \gamma_{\rho} P_{L} P^{\mu \rho} Q_{2}^{-1}\right) u\left(p_{+}\right) \\
& +\bar{u}\left(p_{-}\right)(1 c d)_{q} u\left(p_{+}\right),
\end{aligned}
$$

where the last term linear in $q$ does not contribute to the charge but may contribute to the magnetic moment,

$$
(1 c d)_{q}=\frac{1}{2} P_{R} \gamma_{\sigma}(\not k+\not p) \not P^{\mu \sigma}\left(P Q_{2}\right)^{-1}-\frac{1}{2} \not(\not k+\not p) \gamma_{\rho} P_{L} P^{\mu \rho}\left(P Q_{2}\right)^{-1} .
$$

In summary, leaving aside the $(1 c d)_{q}$ term, we have

$$
\begin{aligned}
\sum_{x=a}^{f}(1 x)_{0}= & +\partial_{\mu}\left\{-m_{W}^{-2}\left[(\not k+\not p)\left(m_{i}^{2} P_{R}+m_{\alpha}^{2} P_{L}\right)-m_{i} m_{\alpha}^{2}\right]\left(P Q_{2}\right)^{-1}\right. \\
& \left.+\gamma_{\sigma}(\not \not L+\not p) \gamma_{\rho} P_{L} P^{\rho \sigma} P^{-1}\right\}+2 \gamma_{\rho} P_{L} P_{\mu}^{\rho} Q_{2}^{-1} .
\end{aligned}
$$

The total derivative can be dropped in regularized loop integrals, so that Fig. 1 contributes to the $F_{1}(0)$ term in Eq. (1) the following:

$$
+e g_{2}^{2} \sum_{\alpha}\left|V_{\alpha i}\right|^{2} \int_{k} \gamma_{\rho} P_{L} P_{\mu}^{\rho} Q_{2}^{-1}=+e g_{2}^{2} \int_{k} \gamma_{\rho} P_{L} P_{\mu}^{\rho} Q_{2}^{-1},
$$

where unitarity of $V$ is used to finish the sum as the integrand is independent of $m_{\alpha}$.

Now we manipulate $i \Pi_{\mu v}(0)$. First of all, $(2 b)$ and $(2 d)$ form a total derivative:

$$
(2 b)+(2 d)=-\left(c_{W}^{2}-s_{W}^{2}\right) \partial_{\mu}\left(k_{v} Q_{2}^{-1}\right) .
$$


Using the shortcuts in Eqs. (18[19), we have

$$
\begin{aligned}
(2 a) & =2 c_{W}^{2}\left[\delta_{\xi}\left(k^{2} g_{\mu \nu}-k_{\mu} k_{v}\right)\left(Q_{1} Q_{2}\right)^{-1}-g_{\mu \nu}(d-1) Q_{1}^{-1}\right], \\
(2 c) & =2 c_{W}^{2}\left[\xi_{W}\left(k^{2} g_{\mu \nu}-k_{\mu} k_{v}\right)\left(Q_{1} Q_{2}\right)^{-1}+2(d-1) k_{\mu} k_{v}\left(Q_{1}\right)^{-2}\right] .
\end{aligned}
$$

The last terms in $(2 a)$ and $(2 c)$ already form a total derivative. In the first terms, we decompose $k^{2}\left(Q_{1} Q_{2}\right)^{-1}=Q_{2}^{-1}+m_{W}^{2}\left(Q_{1} Q_{2}\right)^{-1}$, and then sum judiciously with $(2 f)$ to arrive at the result

$$
\begin{aligned}
(2 a)+(2 c)+(2 f)= & 2 c_{W}^{2}\left\{-(d-1) \partial_{\mu}\left(k_{v} Q_{1}^{-1}\right)+g_{\mu v}\left[Q_{2}^{-1}+m_{W}^{2}\left(Q_{1} Q_{2}\right)^{-1}\right]\right. \\
& \left.+k_{\mu} k_{v}\left[Q_{2}^{-2}-\left(Q_{1} Q_{2}\right)^{-1}\right]-2 k_{\mu} k_{v} Q_{2}^{-2}\right\} \\
= & 2 c_{W}^{2}\left\{\partial_{\mu}\left[k_{v} Q_{2}^{-1}-(d-1)\left(k_{v} Q_{1}^{-1}\right)\right]+m_{W}^{2} Q_{2}^{-1} P_{\mu \nu}\right\} .
\end{aligned}
$$

Thus, using $c_{W}^{2}+s_{W}^{2}=1$, the sum of all graphs is

$$
\sum_{x=a}^{f}(2 x)=\partial_{\mu}\left\{k_{v} Q_{2}^{-1}-2 c_{W}^{2}(d-1)\left(k_{v} Q_{1}^{-1}\right)\right\}+2 m_{W}^{2} Q_{2}^{-1} P_{\mu \nu}
$$

Dropping the regularized total derivative and using Eqs. (3, 5), its contribution to the $F_{1}(0)$ term in Eq. (11) is as follows,

$$
-e g_{2}^{2} \int_{k} Q_{2}^{-1} P_{\mu v} \gamma^{v} P_{L}
$$

which cancels Eq. (28). The vanishing charge is thus established at one loop in $R_{\xi}$ gauge.

\subsection{Magnetic moment}

Moving to the magnetic moment, we follow the computational procedure proposed in sec 2 . Now only the graphs in Fig. 1 contribute. Since $(1 b)$ is quadratic in $q$ when expanding in $q$, it drops out. The next simplest is $(1 f)$. Taking a derivative with respect to $q^{v}$, setting $q=0$ and making it manifestly antisymmetric in $\mu$ and $v$ (denoted by the pair of square brackets below), we have

$$
\left[\partial_{v}^{q}(1 f)_{0}\right]=+\frac{1}{4 m_{W}^{2}} \frac{1}{P^{2} Q_{2}}\left(\left(K_{\mu \nu}^{0}+K_{\mu \nu}^{1}\right)\left(m_{i}^{2} P_{R}+m_{\alpha}^{2} P_{L}\right)-2 m_{i} m_{\alpha}^{2}\left[\gamma_{\mu}, \gamma_{v}\right]\right)
$$

where

$$
\begin{aligned}
K_{\mu \nu}^{0} & =\not p\left[\gamma_{\mu}, \gamma_{v}\right]+\left[\gamma_{\mu}, \gamma_{v}\right] \not p \\
K_{\mu v}^{1} & =\not k\left[\gamma_{\mu}, \gamma_{v}\right]+\left[\gamma_{\mu}, \gamma_{v}\right] \not k .
\end{aligned}
$$

Anticipating that $\left[\partial_{v}^{q}(1 f)_{0}\right]$ is to be sandwiched between the initial and final spinors and noting that the $\not k$ in $K_{\mu v}^{1}$ will yield a $\not p$ upon loop integration, we can apply the limiting EoMs $\not p u=m_{i} u$ after the moment has been isolated. The above is thus reduced to

$$
\left[\partial_{v}^{q}(1 f)_{0}\right] \leftrightharpoons+\frac{1}{m_{W}^{2}} \frac{1}{8 P^{2} Q_{2}}\left(K_{\mu v}^{1}\left(m_{i}^{2}+m_{\alpha}^{2}\right)+2 m_{i}\left[\gamma_{\mu}, \gamma_{v}\right]\left(m_{i}^{2}-m_{\alpha}^{2}\right)\right)
$$


where, from now on, $\leftrightharpoons$ means equality when sandwiched between the spinors or under the loop integration or both. All factors of $P_{L, R}$ are removed in a similar fashion, confirming that the electric dipole moment does not arise at the one loop.

Figs. $(1 c)$ and $(1 d)$ should be treated together for symmetry reasons. There are two sources of terms, one from those explicitly linear in $q$ and the other from the remaining terms (26) when computing the charge. Putting them together and taking the derivative, we have

$$
\begin{aligned}
\partial_{v}^{q}(1 c+1 d)_{0}= & {\left[m_{i}(\not \not d+\not p) \gamma^{\sigma} P_{L}-P_{R} \gamma^{\sigma} m_{i}(\not \not+\not \not)\right]\left[P_{v ; \mu \sigma} Q_{2}^{-1}-k_{v} Q_{2}^{-2} P_{\mu \sigma}\right] P^{-1} } \\
& +\frac{1}{2}\left[\gamma^{\sigma}(\not \not c+\not p) \gamma^{\rho}-\gamma^{\rho}(\not \not t+\not p) \gamma^{\sigma}\right] P_{L} g_{\rho v} P_{\mu \sigma}\left(P Q_{2}\right)^{-1},
\end{aligned}
$$

where

$$
P_{v ; \alpha \beta}=-\frac{1}{2} \partial_{v} P_{\alpha \beta}
$$

Antisymmetrization and applying EoMs yield, after some algebra,

$$
\begin{aligned}
{\left[\partial_{v}^{q}(1 c+1 d)_{0}\right] \leftrightharpoons } & m_{i} K_{\mu \nu}^{2}\left(\frac{\delta_{\xi}}{4 P Q_{1} Q_{2}^{2}}-\frac{1}{4 P Q_{1}^{2} Q_{2}}+\frac{1}{4 P Q_{1} Q_{2}^{2}}\right) \\
& -\left(K_{\mu \nu}^{1}+2 m_{i}\left[\gamma_{\mu}, \gamma_{v}\right]\right) \frac{1}{8 P Q_{1} Q_{2}}
\end{aligned}
$$

where

$$
K_{\mu \nu}^{2}=k_{\mu}\left[\not k, \gamma_{v}\right]+k_{v}\left[\gamma_{\mu}, \not \hbar\right] .
$$

In deriving the above result, we used identities such as

$$
\begin{aligned}
\gamma_{\mu} \not k \gamma_{v}-\gamma_{v} \not k \gamma_{\mu} & =-\frac{1}{2}\left(\not k\left[\gamma_{\mu}, \gamma_{v}\right]+\left[\gamma_{\mu}, \gamma_{v}\right] \not k\right), \\
\not p \not k \gamma_{v}-\gamma_{v} \not k p p & =+\frac{1}{2}\left(\not p\left[\not k, \gamma_{v}\right]+\left[\not k, \gamma_{v}\right] \not p\right) .
\end{aligned}
$$

Now we manipulate $(1 e)$. Taking the derivative, plugging in the propagator $P^{\rho \sigma}$ and doing antisymmetrization, one obtains

$$
\left[\partial_{v}^{q}(1 e)_{0}\right]=-\left(K_{\mu v}^{0}+K_{\mu \nu}^{1}\right) P_{L} \frac{1}{2 P^{2} Q_{1}}+E_{\mu v} P_{L} \frac{\delta_{\xi}}{4 P^{2} Q_{1} Q_{2}}
$$

where, using $p^{2}=m_{i}^{2}$ and the identity

$$
\not k\left[\gamma_{\mu}, \gamma_{v}\right] \not \notin=k^{2}\left[\gamma_{\mu}, \gamma_{v}\right]-2\left(\left[\gamma_{\mu}, \not k k_{v}+\left[\not k, \gamma_{v}\right] k_{\mu}\right)\right. \text {, }
$$

the second term is recast as follows:

$$
\begin{aligned}
E_{\mu v}= & \not k\left((\not k+\not \not)\left[\gamma_{\mu}, \gamma_{v}\right]+\left[\gamma_{\mu}, \gamma_{v}\right](\not k+\not p)\right) \not k \\
= & {\left[(k+p)^{2}-m_{i}^{2}\right] K_{\mu v}^{1}-k^{2} K_{\mu v}^{0} } \\
& +2\left\{k_{\mu}\left(\not p\left[\not k, \gamma_{v}\right]+\left[\not k, \gamma_{v}\right] \not p\right)+k_{v}\left(\not p\left[\gamma_{\mu}, \not k\right]+\left[\gamma_{\mu}, \not k\right] \not p\right)\right\} .
\end{aligned}
$$


Application of the limiting EoMs gives finally

$$
\begin{aligned}
{\left[\partial_{\nu}^{q}(1 e)_{0}\right] \leftrightharpoons } & -\left(K_{\mu \nu}^{1}+2 m_{i}\left[\gamma_{\mu}, \gamma_{v}\right]\right) \frac{1}{4 P^{2} Q_{1}} \\
& +\left(P K_{\mu \nu}^{1}+\left(m_{\alpha}^{2}-m_{i}^{2}\right) K_{\mu \nu}^{1}-2 m_{i} k^{2}\left[\gamma_{\mu}, \gamma_{v}\right]+4 m_{i} K_{\mu \nu}^{2}\right) \frac{\delta_{\xi}}{8 P^{2} Q_{1} Q_{2}} .
\end{aligned}
$$

The graph Fig. 1(a) involves the triple gauge coupling and double gauge boson propagators, making it the most complicated to evaluate. We outline how this is accomplished. Taking the derivative and doing antisymmetrization we have

$$
\left[\partial_{v}^{q}(1 a)_{0}\right]=+P^{-1} \gamma^{\sigma}(\not k+\not p) \gamma^{\rho} P_{L} A_{\rho \sigma ; \mu v},
$$

where

$$
A_{\rho \sigma ; \mu \nu}=-\frac{1}{2} \delta_{\xi} k^{2}\left(\frac{G_{\mu \nu, \rho \sigma}^{0}}{Q_{1}^{2} Q_{2}}+\frac{G_{\mu \nu, \rho \sigma}^{2}}{Q_{1}^{2} Q_{2}^{2}}\right)-\delta_{\xi} \frac{G_{\mu \nu, \rho \sigma}^{2}}{Q_{1}^{2} Q_{2}}-\frac{3}{2} \frac{G_{\mu \nu, \rho \sigma}^{0}}{Q_{1}^{2}}
$$

and

$$
\begin{aligned}
G_{\mu \nu, \rho \sigma}^{0} & =g_{v \sigma} g_{\mu \rho}-g_{v \rho} g_{\mu \sigma}, \\
G_{\mu \nu, \rho \sigma}^{2} & =k_{\mu}\left(g_{v \rho} k_{\sigma}-g_{v \sigma} k_{\rho}\right)-k_{v}\left(g_{\mu \rho} k_{\sigma}-g_{\mu \sigma} k_{\rho}\right) .
\end{aligned}
$$

The contraction with $G^{0}$ is standardized using Eq. (43) into $\left(K_{\mu \nu}^{0}+K_{\mu \nu}^{1}\right) P_{L}$, while the contraction with $G^{2}$ yields, by making use of Eq. (44),

$$
\left(k_{\mu}\left(\not p\left[\not k, \gamma_{v}\right]+\left[\not k, \gamma_{v}\right] \not p\right)+k_{v}\left(\not p\left[\gamma_{\mu}, \not k\right]+\left[\gamma_{\mu}, \not \subset\right] \not p\right)\right) P_{L},
$$

which reduces to $m_{i} K_{\mu \nu}^{2}$ using EoMs. The final form is

$$
\begin{aligned}
{\left[\partial_{\nu}^{q}(1 a)_{0}\right] \leftrightharpoons } & -\left(K_{\mu \nu}^{1}+2 m_{i}\left[\gamma_{\mu}, \gamma_{v}\right]\right)\left(\frac{\delta_{\xi} k^{2}}{8 P Q_{1}^{2} Q_{2}}+\frac{3}{8 P Q_{1}^{2}}\right) \\
& +2 m_{i} K_{\mu \nu}^{2}\left(\frac{\delta_{\xi} k^{2}}{8 P Q_{1}^{2} Q_{2}^{2}}+\frac{\delta_{\xi}}{4 P Q_{1}^{2} Q_{2}}\right) .
\end{aligned}
$$

To summarize our calculation thus far, the terms relevant to the neutrino magnetic moment are given in Eqs. (54/4148/38). The next task is to demonstrate the $\xi_{W}$ cancellation among those terms. We first decompose $\delta_{\xi} k^{2}=Q_{2}-\xi_{W} Q_{1}$ to remove $k^{2}$ from numerators in Eqs. (54/48). The $K_{\mu \nu}^{2}$ terms sum to

$$
\frac{1}{2} m_{i} \delta_{\xi} K_{\mu \nu}^{2}\left(\frac{1}{P Q_{1}^{2} Q_{2}}+\frac{1}{P Q_{1} Q_{2}^{2}}+\frac{1}{P^{2} Q_{1} Q_{2}}\right) .
$$

For any of the three terms in the above, the $k_{\mu}$ and $k_{\nu}$ factors in $K_{\mu \nu}^{2}$ may be simultaneously replaced by $(k+p)_{\mu}$ and $(k+p)_{v}$, because the resulted additional terms, upon the loop integration, will be proportional to

$$
p_{\mu}\left[\not p, \gamma_{v}\right]+p_{v}\left[\gamma_{\mu}, \not p\right]
$$


which vanishes when sandwiched between the initial and final spinors. We make this replacement for the last term in the sum. Using again $\partial_{\mu} Q_{j}^{-1}=-2 k_{\mu} Q_{j}^{-2}$ and $\partial_{\mu} P^{-1}=-2(k+$ p) ${ }_{\mu} P^{-2}$, the sum becomes

$$
\begin{aligned}
& -\frac{1}{4} m_{i} \delta_{\xi}\left(\left[\not k, \gamma_{v}\right] \partial_{\mu}+\left[\gamma_{\mu}, \not h \partial_{v}\right)\left(P Q_{1} Q_{2}\right)^{-1}\right. \\
= & -\frac{1}{4} m_{i} \delta_{\xi}\left[\partial_{\mu}\left(\left[\not k, \gamma_{v}\right]\left(P Q_{1} Q_{2}\right)^{-1}\right)-(\mu \leftrightarrow v)\right]+\frac{1}{2} m_{i} \delta_{\xi}\left[\gamma_{\mu}, \gamma_{v}\right]\left(P Q_{1} Q_{2}\right)^{-1} .
\end{aligned}
$$

The apparently $\xi_{W}$-dependent terms in the sum $\sum_{x=a}^{f}\left[\partial_{v}^{q}(1 x)_{0}\right]$, including the one in Eq. (55) but dropping total derivatives, are collected below:

$$
\begin{aligned}
\sum_{x=a}^{f}\left[\partial_{v}^{q}(1 x)_{0}\right]_{\xi} \leftrightharpoons & m_{i}\left[\gamma_{\mu}, \gamma_{v}\right] \frac{\delta_{\xi}}{4 P Q_{1} Q_{2}} \\
& +\frac{m_{i}}{m_{W}^{2}} \frac{1}{4 P^{2} Q_{2}}\left(K_{\mu \nu}^{1} m_{i}+\left[\gamma_{\mu}, \gamma_{v}\right]\left(m_{i}^{2}-m_{\alpha}^{2}+\xi_{W} m_{W}^{2}\right)\right)
\end{aligned}
$$

The integral of the above second term is simplified using Eq. (63) and EoMs, while the first one is split by $\delta_{\xi}\left(Q_{1} Q_{2}\right)^{-1}=m_{W}^{-2}\left(Q_{1}^{-1}-Q_{2}^{-1}\right)$, so that the $\xi_{W}$ dependence disappears completely from the sum:

$$
\sum_{x=a}^{f}\left[\partial_{v}^{q}(1 x)_{0}\right]_{\xi} \leftrightharpoons m_{i}\left[\gamma_{\mu}, \gamma_{v}\right] \frac{1}{4 m_{W}^{2}}\left(\frac{1}{P Q_{1}}-\frac{1}{P^{2}}\right)
$$

Adding the above with the terms that are explicitly $\xi_{W}$-independent, we obtain the final sum of terms contributing to the neutrino magnetic moment:

$$
\begin{aligned}
\sum_{x=a}^{f}\left[\partial_{v}^{q}(1 x)_{0}\right] \leftrightharpoons & m_{i}\left[\gamma_{\mu}, \gamma_{v}\right] \frac{1}{4 m_{W}^{2}}\left(\frac{1}{P Q_{1}}-\frac{1}{P^{2}}\right)-\left(K_{\mu \nu}^{1}+2 m_{i}\left[\gamma_{\mu}, \gamma_{v}\right]\right) \frac{1}{2 P Q_{1}^{2}} \\
& +\left(\left[m_{W}^{-2}\left(m_{\alpha}^{2}-m_{i}^{2}\right)-2\right] K_{\mu \nu}^{1}-6 m_{i}\left[\gamma_{\mu}, \gamma_{v}\right]\right) \frac{1}{8 P^{2} Q_{1}} .
\end{aligned}
$$

From Eqs. (1458) and the loop integrals defined in the appendix, we obtain for the neutrino $v_{i}$ the magnetic form factor at the vanishing momentum transfer,

$$
F_{2}(0)=-\frac{g_{2}^{2}}{(4 \pi)^{2}} \frac{2 m_{i}^{2}}{m_{W}^{2}} \sum_{\alpha}\left|V_{\alpha i}\right|^{2}\left[\frac{1}{4} I_{1}+J_{2}-\frac{1}{2} K_{2}+\frac{3}{4} J_{1}-\frac{1}{8}\left(2-x_{\alpha}+y_{i}\right) K_{1}\right]
$$

where $I_{1}, J_{1,2}, K_{1,2}$ are functions of the mass ratios $x_{\alpha}=m_{\alpha}^{2} / m_{W}^{2}$ and $y_{i}=m_{i}^{2} / m_{W}^{2}$. This result is indeed manifestly gauge independent in the class of $R_{\xi}$ gauges.

\section{Evaluation in unitary gauge}

Working in unitary gauge means that the limit $\xi_{W} \rightarrow \infty$ is taken before the loop integrals are evaluated. Since $\xi_{W}$ appears exclusively in the propagators of the $W^{ \pm}$gauge bosons, would-be Goldstone bosons $G^{ \pm}$and the ghosts $c^{ \pm}$, only the gauge boson propagator survives the limit,

$$
P_{\mu v}(k) \rightarrow \bar{P}_{\mu v}(k)=\left(g_{\mu v}-m_{W}^{-2} k_{\mu} k_{v}\right) Q_{1}^{-1}
$$


and thus only the pure- $W^{ \pm}$graphs $(a, e)$ in Fig. 1 and $(a, c)$ in Fig. 2 remain. We have presented our calculation in $R_{\xi}$ gauge in a way that can be easily adapted for unitary gauge.

For the charge contribution from Fig. 1 we take the limit $\xi_{W} \rightarrow \infty$ in the integrand (23) where only the total derivative term survives:

$$
[(1 a)+(1 e)]_{0} \rightarrow \partial_{\mu}\left\{\gamma_{\sigma}(\not k+\not p) \gamma_{\rho} P_{L} P^{-1} \bar{P}^{\rho \sigma}\right\}
$$

whose integral vanishes in dimensional regularization. The relevant terms from the photon- $Z$ mixing energy are obtained from Eqs. (30.31), or more readily from Eq.(33),

$$
[(2 a)+(2 c)] \rightarrow \partial_{\mu}\left\{-2 c_{W}^{2}(d-1)\left(k_{v} Q_{1}^{-1}\right)\right\}
$$

whose integral again vanishes. Thus the vanishing of charge at one loop occurs in unitary gauge in a stronger manner: each of the contributions from the proper vertices and the mixing energy vanishes separately.

The magnetic form factor $F_{2}(0)$ can also be obtained from intermediate steps in subsec 3.2 . We can sum Eqs. (54148) and take the limit $\xi_{W} \rightarrow \infty$, or cope directly with the total of all graphs since we know only Figs. 1 $(a, e)$ survive the limit. The latter point can also be seen from explicit results in Eqs.(41(38). Dropping the total derivatives and sending $\xi_{W} \rightarrow \infty$, the potentially $\xi_{W^{-}}$ dependent part of the total in $R_{\xi}$ gauge, Eq. (56), goes exactly to Eq. (57) without additional manipulations. The result in Eq. (59) is thus recovered in unitary gauge.

\section{Summary}

The electromagnetic properties of neutrinos are an interesting topic that is potentially relevant to various astrophysical phenomena and laboratory measurements. Although we know from principles that the charge and dipole moments of a Dirac neutrino are physical quantities and cannot depend on computational methods or the choice of gauge in a consistent theory, this has never been explicitly examined before in a satisfactory manner even at one loop. We have studied this issue in the minimally extended standard model that incorporates neutrinos masses and mixing. We demonstrated at one loop in both $R_{\xi}$ and unitary gauges that the magnetic moment and vanishing charge are indeed gauge-independent quantities. This statement is exact in the sense that it is true for any values of various masses and the lepton mixing matrix as long as the latter is unitary. We have accomplished this by manipulating directly the integrands of loop integrals and employing simple algebraic identities like (43/44/46) and integral relations like (63). We believe this approach is advantageous over the one that handles the results of loop integration, and may be useful in other contexts. Finally, we mention that various approximations to our exact one-loop result for the magnetic moment in Eq. (59) are possible. For instance, when all neutrinos and charged leptons are much lighter than the weak gauge bosons as is the case in SM, we have from the explicit results in the appendix that $F_{2}(0) \approx-\left(3 G_{F} m_{i}^{2}\right) /\left(4 \pi^{2} \sqrt{2}\right)$, where the mixing matrix drops out from the leading term, so that the interaction potential of the neutrino $v_{i}$ of mass $m_{i}$ and spin $\vec{S}$ with an external magnetic moment is, $V \approx\left(3 e G_{F} m_{i}\right) /\left(4 \pi^{2} \sqrt{2}\right) \vec{S} \cdot \vec{B}$, recovering the well-known result in the literature. 


\section{Acknowledgement}

WTH would like to thank the members of theory group at Nankai University for hospitality during a long-term visit when this work was conducted. This work was supported in part by the grant NSFC-11025525 and by the Fundamental Research Funds for the Central Universities No.65030021.

\section{Appendix: some useful integrals}

We list some loop integrals relevant to our evaluation of the magnetic moment. The following relation is used in sec 3 for reduction of terms:

$$
2 p^{2} \int_{k} \frac{k_{\alpha}}{D_{1}^{n_{1}} D_{2}^{n_{2}}}=p_{\alpha} \int_{k}\left(\frac{1}{D_{1}^{n_{1}-1} D_{2}^{n_{2}}}-\frac{1}{D_{1}^{n_{1}} D_{2}^{n_{2}-1}}+\frac{m_{1}^{2}-m_{2}^{2}-p^{2}}{D_{1}^{n_{1}} D_{2}^{n_{2}}}\right),
$$

where $D_{1}=(k+p)^{2}-m_{1}^{2}, D_{2}=k^{2}-m_{2}^{2}$. Using notations in Eq. (20) with $p^{2}=m_{i}^{2}$, the basic integral is

$$
\int_{k}\left(\frac{1}{Q_{1}^{2}}-\frac{1}{P Q_{1}}\right)=\frac{i}{(4 \pi)^{2}} I\left(x_{\alpha}, y_{i}\right)
$$

where $x_{\alpha}=m_{\alpha}^{2} / m_{W}^{2}$ and $y_{i}=m_{i}^{2} / m_{W}^{2}$. For simplicity, we also define the integrals

$$
\begin{aligned}
& \int_{k}\left(\frac{1}{P Q_{1}}-\frac{1}{P^{2}}\right)=\frac{i}{(4 \pi)^{2}} I_{1}\left(x_{\alpha}, y_{i}\right), \\
& \int_{k} \frac{1}{P^{2} Q_{1}}=-\frac{i}{(4 \pi)^{2}} \frac{1}{m_{W}^{2}} J_{1}\left(x_{\alpha}, y_{i}\right), \\
& \int_{k} \frac{1}{P Q_{1}^{2}}=-\frac{i}{(4 \pi)^{2}} \frac{1}{m_{W}^{2}} J_{2}\left(x_{\alpha}, y_{i}\right), \\
& \int_{k} \frac{2 k_{\mu}}{P^{2} Q_{1}}=\frac{i}{(4 \pi)^{2}} \frac{p_{\mu}}{m_{W}^{2}} K_{1}\left(x_{\alpha}, y_{i}\right), \\
& \int_{k} \frac{2 k_{\mu}}{P Q_{1}^{2}}=\frac{i}{(4 \pi)^{2}} \frac{p_{\mu}}{m_{W}^{2}} K_{2}\left(x_{\alpha}, y_{i}\right) .
\end{aligned}
$$

The parametric integral for $I(s, t)$ is

$$
I(x, y)=\int_{0}^{1} d t \ln \left[x t+(1-t)-y t(1-t)-i 0^{+}\right] .
$$

The other functions are related to it by

$$
\begin{aligned}
I_{1}(x, y) & =\ln x-I(x, y), \\
J_{1}(x, y) & =\frac{\partial}{\partial x} I(x, y), \\
J_{2}(x, y) & =J_{1}(1 / x, y / x), \\
K_{1}(x, y) & =y^{-1}\left[I_{1}(x, y)+(1+y-x) J_{1}(x, y)\right], \\
K_{2}(x, y) & =y^{-1}\left[I(x, y)+(1+y-x) J_{2}(x, y)\right] .
\end{aligned}
$$


Note that the singularity at $y=0$ is spurious since the original integrals are smooth there.

The analytic result for $I$ is known for all parameter regions, but we only record it for the case relevant to $\mathrm{SM}$, i.e., for $0 \leq y<x \ll 1$,

$$
I(x, y)=-2-\frac{1}{2 y}(1-x-y) \ln x+\frac{\lambda}{2 y} \ln R,
$$

where

$$
\lambda=\left(1+x^{2}+y^{2}-2 x-2 y-2 x y\right)^{1 / 2}, R=\frac{1+x-y-\lambda}{1+x-y+\lambda} .
$$

The other two functions are

$$
\begin{aligned}
& J_{1}(x, y)=-\frac{1-x+y}{2 y \lambda} \ln R+\frac{1}{2 y} \ln x, \\
& J_{2}(x, y)=-J_{1}(x, y)-\frac{1}{\lambda} \ln R .
\end{aligned}
$$

\section{References}

[1] M. Pospelov and A. Ritz, Annals Phys. 318, 119 (2005) [hep-ph/0504231].

[2] C. Giunti and A. Studenikin, Phys. Atom. Nucl. 72, 2089 (2009) [arXiv:0812.3646 [hep$\mathrm{ph}]]$.

[3] R. Gastmans, S. L. Wu, and T. T. Wu, arXiv:1108.5322 [hep-ph].

[4] R. Gastmans, S. L. Wu, and T. T. Wu, arXiv:1108.5872 [hep-ph].

[5] J. R. Ellis, M. K. Gaillard, and D. V. Nanopoulos, Nucl. Phys. B 106, 292 (1976).

[6] B. L. Ioffe and V. A. Khoze, Fiz. Elem. Chastits. At. Yadra 9, 118 (1978) [Sov. J. Part. Nucl. 9, 50 (1978)].

[7] M. A. Shifman, A. I. Vainshtein, M. B. Voloshin, and V. I. Zakharov, Yad. Fiz. 30, 1368 (1979) [Sov. J. Nucl. Phys. 30, 711 (1979)].

[8] A. I. Vainshtein, V. I. Zakharov, and M. A. Shifman, Usp. Fiz. Nauk 131, 537 (1980) [Sov. Phys. Usp. 23, 429 (1980)].

[9] W. J. Marciano, C. Zhang, and S. Willenbrock, Phys. Rev. D 85, 013002 (2012) [arXiv:1109.5304 [hep-ph]].

[10] H. -S. Shao, Y. -J. Zhang, and K. -T. Chao, JHEP 1201, 053 (2012) [arXiv:1110.6925 [hep-ph]].

[11] W. A. Bardeen, R. Gastmans, and B. E. Lautrup, Nucl. Phys. B 46, 319 (1972). 
[12] B. W. Lee and R. E. Shrock, Phys. Rev. D 16, 1444 (1977).

[13] M. A. B. Beg, W. J. Marciano, and M. Ruderman, Phys. Rev. D 17, 1395 (1978).

[14] W. J. Marciano and A. I. Sanda, Phys. Lett. B 67, 303 (1977).

[15] K. Fujikawa and R. Shrock, Phys. Rev. Lett. 45, 963 (1980).

[16] L. G. Cabral-Rosetti, J. Bernabeu, J. Vidal, and A. Zepeda, Eur. Phys. J. C 12, 633 (2000) [hep-ph/9907249].

[17] M. Dvornikov and A. Studenikin, Phys. Rev. D 69, 073001 (2004) [hep-ph/0305206].

[18] M. Dvornikov and A. Studenikin, J. Exp. Theor. Phys. 99, 254 (2004) [hep-ph/0411085].

[19] R. Z. Roskies, M. J. Levine and E. Remiddi, Adv. Ser. Direct. High Energy Phys. 7, 162 (1990).

[20] A. Czarnecki and B. Krause, Nucl. Phys. Proc. Suppl. 51C, 148 (1996) [hep-ph/9606393].

[21] A. Czarnecki and B. Krause, Acta Phys. Polon. B 28, 829 (1997) [hep-ph/9611299]. 